sisches Ministerium für Umwelt, Energie, Landwirtschaft und Verbraucherschutz, Wiesbaden) zum gewässerbezogenen Teil des Symposiums über. Dabei ging sie zunächst auf die Neuerungen des WHG ein, das ebenso wie das BNatSchG n. F. zum 1. März 2009 in Kraft trete. Die bisher in den meisten Landeswassergesetzen verankerte ,gehobene Erlaubnis“" werde nunmehr auch auf Bundesebene geregelt ( $\$ 15$ WHG n.F.). Gleichzeitig blieben die ,alten Rechte und Befugnisse" bestehen $(\$ 20)$, ebenso wie das Bewirtschaftungsermessen ( $\$ 12$ Abs. 2). Neu in das WHG eingefügt seien Regelungen zur Mindestwasserführung, Gewässerdurchgängigkeit und Wasserkraftnutzung (vgl. \$S 33 bis 35), die bisher schon teilweise in einigen Landeswassergesetzen verankert waren. In Hessen überlege man derzeit, welche gesetzlichen Anpassungen bis zum Inkrafttreten des neuen WHG zu leisten sind. Dabei wolle man von den bundesrechtlichen Vorgaben möglichst nicht zu viel abweichen. Allerdings müsse man auch darauf achten, dass die bisherigen hessischen Standards nicht unterschritten würden.

Erwartungen an das neue Wasserrecht unter besonderer Berücksichtigung naturschutzrechtlicher Vorgaben für wasserrechtliche Entscheidungen wurden anschließend von RA Klaus-D. Fröhlich (Kanzlei WKLP, Bonn) formuliert. Dabei ging er u.a. auf die schwierige Abgrenzung zwischen naturnahem Gewässerausbau und ökologischer Gewässerunterhaltung ein, bei der auch die Neuregelung der Gewässerunterhaltung in \$39 WHG n. F. nicht weiterhelfe Diese sei zwar detallierter gefasst als bisher, allerdings wirke die Vorschrift zu statisch und trage dem Problem dynamischer Gewässerentwicklung durch Initialmaßnahmen
(z.B. durch das Einbringen von Totholz mit der Folge einer einhergehenden starken Mäandrierung des Gewässers) nicht Rechnung. Im Hinblick auf das Verhältnis von Wasserrecht und Naturschutzrecht machte der Referent deutlich, dass es sich hierbei um zwei eigenständige Rechtsbereiche handele, die trotz der nachzuweisenden Tendenz der „Ökologisierung“ des WHG konfligieren könnten. Das Erfordernis einer FFH-Verträglichkeitsprüfung im Rahmen wasserrechtlicher Entscheidungen bestehe auf Grund von $\$ 34$ BNatSchG fort, auch wenn die betreffende Regelung des $\$ 6$ Abs. 2 WHG a.F. nicht in $\$ 12$ WHG n.F. aufgenommen worden sei.

Das Symposium wurde abgerundet durch einen Vortrag von RA Dirk Teßmer (Frankfurt a. M.) über Bewertungen und Handlungsansätze aus dem Verbandsbeteiligungsrecht. Darin befasste er sich im Kern mit der Frage, wie die besprochenen materiellen Inhalte des BNatSchG in der Praxis durchgesetzt werden könnten. Das neue BNatSchG enthalte insoweit kaum substanzielle Änderungen. $\mathrm{Zu}$ erwähnen seien allerdings die neu hinzugekommenen Mitwirkungsrechte anerkannter Naturschutzvereinigungen bei der Erteilung von Befreiungen von Geboten und Verboten zum Schutz von geschützten Meeresgebieten im Bereich der deutschen AWZ und des Festlandssockels ( $\$ 63$ Abs. 1 Nr. 2 BNatSchG n.F.). De lege ferenda plädierte er dafür, die naturschutzrechtlichen Verbandsbeteiligungs- und -kontrollrechte auf planfeststellungsersetzende Bebauungspläne, Befreiungen in Landschaftsschutzgebieten, Ausnahmen vom gesetzlichen Biotopschutz und artenschutzrechtliche Befreiungen auszudehnen.
DOI: 10.1007/s10357-009-1805-0

\section{Beschlüsse und anhängige Klagen vor dem Europäischen Gerichtshof}

Klage eingereicht am 25.9.2009-

Kommission/Französische Republik (C-383/09)

Klagegründe und wesentliche Argumente

Die Europäische Kommission rügt mit ihrer Klage, dass die Beklagte nicht, wie es Art. 12 der Richtlinie 92/43/EWG verlange, ein strenges Schutzsystem für die Art Cricetus cricetus (Europäischer Hamster) im Elsass, das das natürliche Verbreitungsgebiet dieser Art in Frankreich darstelle, eingeführt habe.

Die Zählungen der Baue des Tieres hätten einen erheblichen Rückgang der Bestände in den letzten Jahren aufgezeigt, da die Zahl der Baue von 1167 im Jahr 2001 auf nur 161 im Jahr 2007 zurückgegangen sei. Unter diesen Voraussetzungen sei die Art, die durch für sie nachteilige Landwirtschaftspraktiken und Siedlungsdruck bedroht werde, kurzfristig von völligem Aussterben bedroht.

Die Kommission erkennt in ihrer Klageschrift an, dass die Beklagte die Probleme durch den Erlass von Maßnahmen sowohl in Bezug auf Raumordnung als auch auf die landwirtschaftlichen Praktiken berücksichtigt habe, doch seien diese Maßnahmen völlig unzureichend.

Zum einen deckten die drei vorrangigen Aktionsgebiete, bei denen es sich um die Gebiete handele, auf die sich die Anstrengungen zur Rettung der Art im Wesentlichen konzentriere, nur einen sehr

Jochen Schumacher,

Tübingen, Deutschland kleinen Teil des Gebiets ab, das den natürlichen Lebensraum dieser Art darstelle, da sich zwei Drittel der bestehenden Baue außerhalb dieser Gebiete befänden, die wiederum nur 2\% der für den Europäischen Hamster günstigen Flächen darstellten. Um eine zweckdienliche gebietsmäßige Abdeckung der Maßnahmen zum Schutz dieser Art zu gewährleisten, müsse zumindest das Vorkommen des Europäischen Hamsters im Jahr 1990 und nicht im Jahr 2000 als Bezugswert genommen werden.

Zum anderen seien die Schutzmaßnahmen selbst sehr unzulänglich. Die Kommission rügt in diesem Zusammenhang insbesondere das Fehlen einer klaren Regelung in Bezug auf den Raum, in dem der Hamster wieder heimisch werden solle. Die nationale Verwaltung verfüge nämlich über einen zu großen Ermessensspielraum bei der Gewährung von Ausnahmen für die Ausarbeitung von Raumordnungsvorhaben in den von den Hamstern bewohnten Gebieten, und es herrsche große Unsicherheit in Bezug auf die für den Schutz dieser Art ergriffenen Ausgleichsmaßnahmen.

Beschluss des Gerichtshofs vom 23. September 2009 Complejo Agrícola, SA/Kommission der Europäischen Gemeinschaften, Königreich Spanien (C-415/08 P)

Rechtsmittel gegen den Beschluss des Gerichts erster Instanz (Erste Kammer) vom 14. Juli 2008, Complejo Agrícola/Kommission (T-345/06), mit dem das Gericht die Klage auf teilweise Nichtigerklärung von Art. 1 und Anhang 1 der Entscheidung 2006/613/EG der Kommission vom 19. Juli 2006 zur Festlegung der Liste der Gebiete von gemeinschaftlicher Bedeutung in der mediterranen biogeografischen Region gemäß der Richtlinie 92/43/EWG des Rates (AB1. L 259, S. 1), soweit darin das Gebiet namens „Acebuchales de la Campiña Sur de Cádiz“, in dem sich ein der Rechtsmittelführerin gehörender Landwirtschaftsbetrieb befindet, als Gebiet von gemeinschaftlicher Bedeutung in der mediterranen biogeografischen Region ausgewiesen wird, als unzulässig abgewiesen hat. 